\title{
A REMARK ON ALGEBRAS OF MATRICES
}

\author{
WINSTON M. SCOTT
}

1. Introduction. Let $\mathfrak{A}$ denote a matrix algebra, with unit element, over an algebraically closed field $K$. We shall assume that $\mathfrak{A}$ is in reduced form, that is, that $\mathfrak{A}$ is exhibited with only zeros above the main diagonal, with irreducible constituents of $\mathfrak{A}$ in the main diagonal, and that $\mathfrak{A}$ is expressible as the direct sum of its radical and a semisimple subalgebra which latter has nonzero components only in the irreducible constituents of $\mathfrak{A}$ :

$$
\mathfrak{A}=\left(\begin{array}{cccc}
\mathfrak{c}_{11} & \cdots & \ldots & \cdot \\
\mathfrak{夭}_{21} & \mathfrak{c}_{22} & \ldots & \cdot \\
\cdot & \cdot & \cdots & \cdot \\
\mathfrak{c}_{t 1} & \mathfrak{c}_{t 2} & \cdots & \mathfrak{c}_{t t}
\end{array}\right),
$$

the $\mathfrak{E}_{i i}$ denoting irreducible constituents; further $\mathfrak{U}=\mathfrak{H}^{*}+\mathfrak{N}$ where $\mathfrak{N}$ is the radical of $\mathfrak{A}$ and

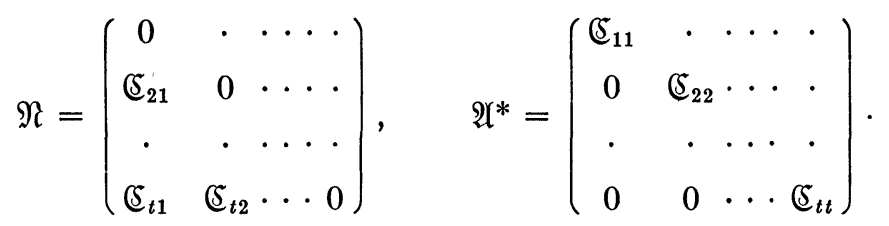

As a part of $\mathfrak{A}, \mathfrak{E}_{i j}$ forms an additive group or module of matrices upon which $\mathfrak{A}$, itself considered as a module, is homomorphically mapped. We shall consider $\mathfrak{E}_{i j}$ as a matrix module with $\mathfrak{A}$ as both left and right operator system. For a matrix $A$ of $\mathfrak{A}$, we shall use the notation $C_{i j}(A),(j \leqq i, i=1,2, \cdots, t)$, to denote the parts of $A$,

$$
A=\left(\begin{array}{cccc}
C_{11}(A) & \cdot & \cdots & 0 \\
C_{21}(A) & C_{22}(A) & \cdots & \cdot \\
\cdot & \cdot & \cdots & \cdot \\
C_{t 1}(A) & \cdot & \cdots & C_{t t}(A)
\end{array}\right)
$$

Let $B$ be any element of $\mathfrak{A}$, and let $B^{*}$ be the component of $B$ in the semisimple subalgebra $\mathfrak{A}^{*}$. We define $B$ as a left and as a right operator of $C_{i j}(A)$ by the relations below, using $\circ$ to distinguish this operation from ordinary matrix multiplication

Received by the editors June 15, 1942. 


$$
\begin{aligned}
& B \circ C_{i j}(A)=C_{i i}(B) \cdot C_{i j}(A)=C_{i j}\left(B^{*} A\right), \\
& C_{i j}(A) \circ B=C_{i j}(A) \cdot C_{j j}(B)=C_{i j}\left(A B^{*}\right) .
\end{aligned}
$$

We shall indicate that a matrix module has $\mathfrak{A}$ as both right and left operator system by calling the module an ( $\mathfrak{A}, \mathfrak{A})$ module. Under (4), $\mathfrak{S}_{i j}$ is a simple $(\mathfrak{A}, \mathfrak{A})$ module. Moreover, each $\mathfrak{S}_{i j}$ is either a 0 -part or there exist elements in $\mathfrak{A}$ such that the corresponding $C_{i j}$ have any arbitrary components from $K$. We shall call the $\mathfrak{S}_{i j}$ simple parts of $\mathfrak{A} .^{1}$

2. The basic theorem. Professor R. Brauer, in a recent paper, ${ }^{2}$ has proved a theorem which has a great many applications and among other things includes the Jordan-Hölder Theorem as a special case.

BASIC THEOREM. Let $G$ and $H$ be two groups, with finite composition lengths, for both of which a given set $\theta$ is the operator set. Let

$$
G=G_{0} \supset G_{1} \supset G_{2} \supset \cdots \supset G_{r}=(1)
$$

be a composition series of $G$, and

$$
H=H_{0} \supset H_{1} \supset H_{2} \supset \cdots \supset H_{s}=(1)
$$

be a composition series of $H$. If $\theta$ is a homomorphism which maps $H$ upon a normal subgroup $H^{*}$ of $G, H^{*} \subseteq G$, then one can choose complete residue systems $P_{\rho}$ of $G_{\rho-1}\left(\bmod G_{\rho}\right)$ and $Q_{\sigma}$ of $H_{\sigma-1}\left(\bmod H_{\sigma}\right)$, $(\rho=1,2, \cdots, r ; \sigma=1,2, \cdots, s)$, such that

(a) either $\theta$ maps $Q_{\sigma}$ on a $P_{\rho}$ in a $(1-1)$ manner and $G_{\rho-1} / G_{\rho}$ $=H_{\sigma-1} / H_{\sigma}$, or $\theta$ maps $Q_{\sigma}$ on 1 , and

(b) each $P_{\rho}$ is the image of at most one $Q_{\sigma}$..

Here, in our application of the basic theorem, $H$ will be mapped on the whole group $G$ and, consequently, statement (b) of the theorem may be sharpened to "Each $P_{\rho}$ is the image of exactly one $Q_{\sigma}$. "

3. An application of the basic theorem. We shall first prove:

ThEOREM 1. A composition series

$$
\mathfrak{A}=\mathfrak{A}_{0} \supset \mathfrak{A}_{1} \supset \mathfrak{A}_{2} \supset \ldots \supset \mathfrak{A}_{m}
$$

of a matrix algebra $\mathfrak{A}$ considered as an $(\mathfrak{A}, \mathfrak{N})$ module is also a composi-

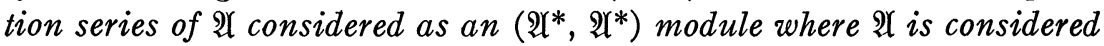
as the direct sum of $\mathfrak{A}^{*}$, a semisimple subalgebra, and $\mathfrak{N}$, the radical of $\mathfrak{A}$.

\footnotetext{
${ }^{1}$ For a further study of simple parts, see W. M. Scott, On matrix algebras over an algebraically closed field, Ann. of Math. vol. 43 (1942) pp. 147-161.

${ }^{2} \mathrm{R}$. Brauer, On sets of matrices with coefficients in a division ring, Trans. Amer. Math. Soc. vol. 49 (1941) pp. 502-548.
} 
Proof. In $\mathfrak{A}_{i-1} / \mathfrak{A}_{i}$ we consider all residue classes $\left\langle A^{(i-1)}\right\rangle$. Select the classes different from $\langle 0\rangle$ which have representatives belonging to $^{3}$ a highest power of the radical $\mathfrak{N}$, say $\mathfrak{N}^{\rho}$. These together with the $\langle 0\rangle$ class form an admissible subgroup with elements of $\mathfrak{A}$ as operators on both left and right (that is, as ( $\mathfrak{A}, \mathfrak{U})$ operators), for if we have $\left\langle A^{(i-1)}\right\rangle$ such that $A^{(i-1)}$ belongs to $\mathfrak{N}^{\rho}$, then

$$
A\left\langle A^{(i-1)}\right\rangle B=\left\langle A A^{(i-1)} B\right\rangle
$$

has the representative $A A^{(i-1)} B$ which is contained in $\mathfrak{l}^{\rho}$. Then this admissible subgroup must coincide with the whole factor group $\mathfrak{U}_{i-1} / \mathfrak{A}_{i}$ since $\mathfrak{A}_{i-1} / \mathfrak{A}_{i}$ is simple. We can conclude, then, that every class in $\mathfrak{A}_{i-1} / \mathfrak{A}_{i}$ has a representative which belongs to $\mathfrak{N}^{\rho}$.

Now let $\left\langle A^{(i-1)}\right\rangle$ be a class with $A^{(i-1)}$ belonging to $\mathfrak{R}^{\rho}$. We have that for $N \in \mathfrak{N}$

$$
N\left\langle A^{(i-1)}\right\rangle=\left\langle N A^{(i-1)}\right\rangle
$$

has a representative in $\mathfrak{N}^{\rho+1}$. But we have selected all classes different from $\langle 0\rangle$ belonging to a highest power which was $\mathfrak{N}^{\rho}$. Then the class $\left\langle N A^{(i-1)}\right\rangle$ must be the 0 -class. Therefore, we have that for $A \in \mathfrak{A}$, $A=A^{*}+N, A^{*} \in \mathfrak{Y}^{*}$, and $N \in \mathfrak{N}$,

$$
\begin{aligned}
A\left\langle A^{(i-1)}\right\rangle & =\left\langle A \cdot A^{(i-1)}\right\rangle \\
& =\left\langle A^{*} \cdot A^{(i-1)}+N \cdot A^{(i-1)}\right\rangle \\
& =\left\langle A^{*} \cdot A^{(i-1)}\right\rangle \\
& =A^{*}\left\langle A^{(i-1)}\right\rangle,
\end{aligned}
$$

and our theorem is proved.

Now considering the simple part $\mathfrak{C}_{i j}$ as an $\left(\mathfrak{P}^{*}, \mathfrak{U}^{*}\right)$ module (as we may, since $\left.\mathfrak{A}^{*} \subset \mathfrak{A}\right)$, we have that $A \rightarrow C_{i j}(A)$ is an $\left(\mathfrak{A}^{*}, \mathfrak{I}^{*}\right)$ homomorphism so that by our basic theorem $\mathfrak{E}_{i j}$ is $\left(\mathfrak{I}^{*}, \mathfrak{I}^{*}\right)$ isomorphic to a composition factor group of $\mathfrak{A}$. But the operators of the system $\mathfrak{A}$ on $\mathfrak{S}_{i j}$ and on the factor group are equivalent by (4) and Theorem 1 to operators of the system $\mathfrak{A}^{*}$. From this we have that $\mathfrak{E}_{i j}$ is also $(\mathfrak{A}, \mathfrak{U})$ isomorphic to the composition factor group.

Thus we have, by the basic theorem, a proof of the following theorem.

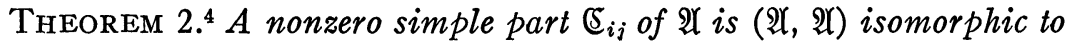

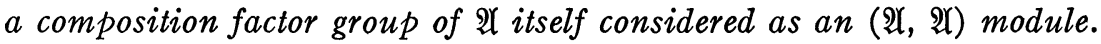

WASHington, D. C.

${ }^{3}$ For a definition of belonging to as used here, see C. Nesbitt, On the regular representations of algebras, Ann. of Math. vol. 39 (1938) pp. 634-658.

${ }^{4}$ For a direct proof of this theorem, see W..M. Scott, op. cit. 